\section{Obere Atemwege}

Helga Peter

Marburg, Deutschland

\section{Definition}

Bezeichnung für die Atemwege oberhalb des Kehlkopfes: Rachen, Nase und Mund; siehe auch $\triangleright$,Pharynx“.

\title{
Synonyme
}

Extrathorakale Atemwege 